\title{
Learning to rank for why-question answering
}

\author{
Suzan Verberne $\cdot$ Hans van Halteren • Daphne Theijssen • \\ Stephan Raaijmakers • Lou Boves
}

Received: 7 October 2009/Accepted: 18 May 2010/Published online: 5 June 2010

(C) The Author(s) 2010. This article is published with open access at Springerlink.com

\begin{abstract}
In this paper, we evaluate a number of machine learning techniques for the task of ranking answers to why-questions. We use TF-IDF together with a set of 36 linguistically motivated features that characterize questions and answers. We experiment with a number of machine learning techniques (among which several classifiers and regression techniques, Ranking SVM and $S V M^{\text {map }}$ ) in various settings. The purpose of the experiments is to assess how the different machine learning approaches can cope with our highly imbalanced binary relevance data, with and without hyperparameter tuning. We find that with all machine learning techniques, we can obtain an MRR score that is significantly above the TF-IDF baseline of 0.25 and not significantly lower than the best score of 0.35 . We provide an in-depth analysis of the effect of data imbalance and hyperparameter tuning, and we relate our findings to previous research on learning to rank for Information Retrieval.
\end{abstract}

Keywords Learning to rank - Question answering · Why-questions

\section{Introduction}

In the field of question answering (QA), abundant attention has been paid to questions with factive answers such as factoids and definitions. For a long time, the problem of whyquestions was largely neglected by researchers in the QA field. The main reason is that explanation-type questions are considered one of the most complex question types (Maybury 2002) and they require a different treatment than questions with factive answers:

\footnotetext{
S. Verberne $(\bowtie) \cdot$ H. van Halteren $\cdot$ L. Boves

Centre for Language and Speech Technology, Radboud University, Nijmegen, Netherlands

e-mail: s.verberne@let.ru.nl

D. Theijssen

Department of Linguistics, Radboud University, Nijmegen, Netherlands

S. Raaijmakers

TNO Information and Communication Technology, Delft, Netherlands
} 
Answers to why-questions cannot be stated in a single phrase but they are passages of text that contain some form of (possibly implicit) explanation (Verberne et al. 2007).

In previous work (Verberne et al. 2010), we described a system for why-QA that consists of an off-the-shelf passage retrieval engine (Lemur ${ }^{1}$ ), and a ranking module that uses a set of features extracted from the question and each of the candidate answers. Until now, we have mainly focused on improving the ranking performance of our system by adapting and expanding the feature set used for ranking. This has led to a set of 37, mostly linguistically motivated, features representing the degree of overlap between a question and each of its candidate answers. We have experimented with a genetic algorithm and with logistic regression for finding the optimal weights for combining the 37 features (Verberne et al. 2008, 2010).

In the current paper, we aim at finding the optimal ranking function for our feature set to be applied in the ranking module to the set of candidate answers. We evaluate a number of learning-to-rank techniques (Liu 2009) in their ability of ranking the answers in our data set. The problem of answering why-questions is of interest because the length and the complexity of the answers make it an interesting case study for answer ranking with the use of linguistically motivated features.

The problem of learning to rank has gained attention in the field of Information Retrieval (IR) since 2005. It has been boosted by the ongoing development of the LETOR benchmark data set (Liu et al. 2007). Until now, most learning-to-rank research has been directed at developing new techniques and evaluating them on the LETOR data collections. This has resulted in a good understanding of the performance of a range of ranking techniques for this specific data set. However, it is not yet known to what extent their performances will change for other data sets. This paper is a step towards understanding to what extent the results do generalize to other data and applications.

Learning-to-rank experiments are meaningful for applications that produce a ranked list of items (documents, entities, answers, etc.) that are described by a set of features and a class label according to which they can be ranked. In IR applications, the class label refers to the item's relevance. In the case of QA, relevance is generally defined as a binary variable (Voorhees et al. 2000). On the other hand, all operational QA systems still present a ranked list of answer candidates for each individual input question (Shen et al. 2005). For our system for why-QA, we also use binary relevance labeling while aiming at a ranked result list. Although we found that it is to some extent possible to label the answers to whyquestions on a multi-level relevance scale, we decided to treat answer relevance as a binary variable (see Sect. 3.3). This means that our ranking function needs to induce a ranked list from binary relevance judgments. ${ }^{2}$

A second challenge that we face in learning to rank our data is the imbalance between positive and negative instances in the training set: There tend to be much more incorrect than correct answers (Usunier et al. 2004). This is not unique for QA data (in document retrieval, for example, the number of irrelevant documents is also much larger than that of relevant ones) but we will see that this imbalance plays a role in ranking the answers in our data collection.

In this paper, we evaluate the following techniques for the task of learning a ranking for why-answers: Naïve Bayes, Support Vector Classification, Support Vector Regression, Logistic Regression, Ranking SVM, SVM map and a Genetic Algorithm. Following the

\footnotetext{
${ }^{1}$ See http://www.lemurproject.org.

${ }^{2}$ In ranking binary relevance data, the goal is to rank the correct answers higher than the incorrect answers. There is no evaluation of the ranking among the (in)correct answers themselves.
} 
learning-to-rank literature (Liu 2009), we consider three different approaches to learning to rank: (1) the so-called pointwise approach, in which candidate answers are classified individually (over all questions), (2) the pairwise approach, in pairs of two candidate answers to the same question are classified, and (3) the listwise approach, in which the complete ranking of all candidate answers to the same question is optimized.

We will discuss the performance of each of these three approaches on our data while evaluating the machine learning techniques mentioned above. Some of these techniques require tuning of hyperparameters, others do not. We will pay special attention to the effects of data imbalance and hyperparameter tuning in the performance of the techniques.

In the remainder of this paper, we will use the term 'answer cluster' to refer to the set of candidate answers to one question. We assume a set-up in which a list of candidate answers is retrieved by a retrieval engine. Learning to rank is the task of learning the optimal ranking for the answers within each cluster.

This paper is organized as follows: in Sect. 2, we discuss related work on QA research, learning to rank and the problem of imbalanced data. In Sect. 3 we describe the resources that we use for our experiments and we specify the characteristics of the data used in our experiments. An overview of the experiments that we conducted is in Sect. 4. The results are presented in Sect. 5, followed by a detailed discussion of the results in Sect. 6. Section 7 contains our conclusions.

\section{Related work}

In Sect. 2.1, we give a summary of QA research, the role of why-questions in QA and learning-to-rank experiments for the purpose of QA. In Sect. 2.2, we present a brief overview of the learning-to-rank approaches in the literature. In Sect. 2.3, we discuss the challenge of classifying imbalanced data, which is an important aspect of our learning problem.

\subsection{Question answering and the position of why-questions}

Question answering research emerged in the field of Information Retrieval in the mid1990s. From 1999 to 2007, the TREC-QA track ${ }^{3}$ has encouraged the development and evaluation of open-domain QA systems, with the use of common evaluation measures. In the first years of the TREC-QA track, different question types were included in one and the same task. The 1999 QA track contained 200 questions, only two of which were whyquestions; all other questions were factoids (asking after who, where, how many, etc.; Voorhees 2000). From 2002 onwards, why-questions were no longer included in the track's main task (Voorhees 2003).

According to the 2002 overview paper by Maybury (2002), why-questions are one of the most complex question types. This is mainly because the answers to why-questions are not named entities (which are in general clearly identifiable), but text passages giving a (possibly implicit) explanation (Verberne et al. 2007). Recently, research by Higashinaka and Isozaki (2008) has been directed at developing and evaluating QA systems for answering Japanese why-questions (why-QA). For English why-questions, we have in previous work developed an approach that combines bag-of-words retrieval techniques with linguistic and structural knowledge (Verberne et al. 2010). The current paper will

\footnotetext{
${ }^{3}$ See http://www.trec.nist.gov/data/qamain.html.
} 
continue this line of work with learning-to-rank experiments for our set of structural and linguistic features.

Until now, not much research has been directed at learning-to-rank experiments for the purpose of optimizing QA systems. In 2004, Usunier et al. are the first to apply learning-torank techniques to QA data: they experiment with AdaBoost and RankBoost on a set of 250 questions. Surdeanu et al. (2008) adopted the Ranking Perceptron approach of Shen and Joshi (2005) for learning to rank in the context of a large QA collection.

\subsection{Learning-to-rank approaches}

Most approaches to learning to rank consider the problem as a case of supervised learning. All instances (the items to be ranked) are assigned a (binary or ordinal) score representing their relevance as determined by an independent judgment process; this score is considered as the ground truth. In the training stage, a ranking function is learned based on the set of feature vectors with their ground truth labels. In the testing stage, the function is applied to new sets of items in order to generate a ranked order. Learning a ranking function is not a trivial task. In Liu (2009), many approaches to learning to rank are discussed.

Approaches to learning a ranking from a set of labeled instances can be divided in three categories: (1) learning to classify the instances according to their (binary or ordinal) label irrespective of the clustering of the answers ${ }^{4}$ (pointwise approach), (2) classifying pairs of correct and incorrect answers for their mutual order and optimize the proportion of correctly ordered pairs (pairwise approach), or (3) optimizing a cost function for the ordering of answers within one answer cluster (listwise approach). In the remainder of this section, we discuss the three approaches in more detail.

\subsubsection{The pointwise approach}

In the pointwise approach, the clustering of answers per question is ignored in the training stage: The task of the classifier is to learn whether an instance should be classified as relevant or irrelevant, irrespective of the answer cluster it belongs to. Relations between the candidate answers to the same question are ignored. A ranked answer list can then be induced by letting the classifier assign a score to each instance in the test set, expressing the probability that it should be classified as relevant, and then ordering the answers per question according to these scores (ordinal sort) (Burges et al. 2005). Techniques that can be applied in this approach are classifiers (such as Naïve Bayes and Support Vector Classification) and regression techniques (such as Logistic Regression and Support Vector Regression; Cossock and Zhang 2006; Herbrich et al. 2002).

In the literature, the pointwise approach is considered the weakest of the three learningto-rank approaches, because it ignores the clustering of instances per query. This especially leads to problems in situations where the number of answers varies largely for different queries. ${ }^{5}$ Moreover, pointwise approaches do not take into account the position of each answer in a ranked list. As a result of that, candidate answers that are in the bottom part of the result list receive the same attention as the top-ranked candidates while they are relatively less important for the overall system performance (Liu 2009).

\footnotetext{
${ }^{4}$ We use the word 'answers' here because that is the type of data we are working with in our QA experiments. In learning-to-rank experiments, 'answers' can be any type of items to be ranked.

5 This type of imbalance was not relevant in our study since we ranked 150 answers for all questions.
} 


\subsubsection{The pairwise approach}

An alternative way of learning a ranking for a list of answers is to classify pairs of relevant and irrelevant answers within one cluster for their mutual order and optimizing the proportion of correctly ordered answers. This learning principle is called 'pairwise preference learning', and was introduced by Joachims (2002), who proposed the learning algorithm Ranking SVM based on this principle. Other pairwise algorithms are RankNet (Burges et al. 2005) and RankBoost (Freund et al. 2003). Pairwise preference learning is studied in more detail in Furnkranz and Hullermeier (2003) and is applied to several ranking problems such as combining rankings from multiple retrieval systems in Carterette and Petkova (2006).

Pairwise approaches are considered more powerful than pointwise approaches because they consider pairs of instances from the same cluster and do not take into account unrelated instances (answers to other queries). Furthermore, pairwise approaches tend to give better results on the LETOR benchmark data than pointwise approaches, although the differences are small and not always significant (Liu 2009). ${ }^{6}$

\subsubsection{The listwise approach}

The third, more recently developed approach to learning to rank answers is the listwise approach, in which a cost function for the ordering of answers within one answer cluster is optimized (Xia et al. 2008). There are two subtypes of listwise approaches (Liu 2009). The first takes into account the relevance labels of all instances within one cluster and optimizes the instance order for an IR evaluation measure such as Mean Average Precision (MAP), Mean Reciprocal Rank (MRR) or Normalized Discount Cumulative Gain (nDCG). Examples of techniques that optimize for IR evaluation measures are $S V M^{m a p}$ (Yue et al. 2007) and AdaRank (Xu et al. 2007). The second type of listwise learning [e.g. ListNet (Cao et al. 2007)] takes lists of ranked items as training data, and optimizes for the difference between this ground truth ranking and the hypothesized ranking. Listwise techniques are considered promising because they already reach scores similar to or better than pairwise techniques while they have been developed more recently (Liu 2009).

In Verberne et al. (2008) we implemented a listwise ranking approach using a genetic algorithm that optimizes answer ranking for MRR. Genetic algorithms have been applied to learning-to-rank problems and other retrieval optimization problems by several researchers in the field (Trotman 2004; Yeh et al. 2007; Tiedemann 2007). The ranking performance can be defined and implemented in the so-called fitness function in different ways. In Fan et al. (2004), a number of fitness functions that are derived from ranking evaluation measures (such as MAP) are compared for their effectiveness. The conclusion was that the best results are obtained when the algorithm is optimized for the same measure as it is evaluated on.

\subsection{The problem of imbalanced data}

As mentioned in Sect. 1, class imbalance is a challenge in many learning-to-rank tasks, also in ranking QA data (Usunier et al. 2004). This especially holds for pointwise techniques because the imbalance hampers the optimization process: If $98 \%$ of the instances in

\footnotetext{
${ }^{6}$ All experimental results on the LETOR data can be downloaded from http://www.research.microsoft. com/en-us/um/beijing/projects/letor/.
} 
the training set have been labeled irrelevant (or incorrect), then classifying all instances as incorrect gives an accuracy of $98 \%$.

This problem has been acknowledged by many researchers in the machine learning field (Japkowicz and Stephen 2002; Usunier et al. 2004; Akbani et al. 2004; Tang et al. 2009). Because SVMs are very popular for all sorts of classification tasks, much work on tackling the problem of imbalanced data is focused on making SVMs robust to imbalance. In the literature, three solutions for curing problematic class imbalances for classifiers are discussed: undersampling the majority class, oversampling the minority class and costmodifying according to the same ratio as the class balance. In general, the latter approach gives the best results for various classifiers (Japkowicz and Stephen 2002; Tang et al. 2009).

Class imbalance causes fewer problems for regression techniques than for classifiers. In regression models, the so-called 'intercept' value moves the outcome of the regression function towards the bias in the data. If the class imbalance is not too extreme, the intercept can be adapted so that the regression function is robust against it (Owen 2007).

Pairwise approaches are less sensitive to class imbalance than pointwise approaches. The reason is that they classify pairs of correct and incorrect answers from the same cluster, thereby balancing the training data. For listwise approaches, data imbalance is not an issue since these techniques are not classification-based but they optimize for instance order directly.

\section{Data and system set-up}

In this section, we present the background of our learning to rank task in terms of the resources that we use for development and evaluation (1), the set-up of our QA system (2), the ground truth labeling that we apply to the data (3) the features that we extract from the data (4), and our evaluation set-up (5).

\subsection{Resources}

Our passage database is extracted from the Wikipedia INEX 2006 corpus (Denoyer and Gallinari 2006). This corpus consists of all 659,388 articles extracted from the online Wikipedia in the summer of 2006, converted to XML format. Before indexing the corpus, we segmented all Wikipedia articles into passages. We used a semi-fixed passage size of 500-600 characters (excluding all XML markup) with an overflow to 800 for the purpose of completing sentences. ${ }^{7}$ We created passage overlap by starting each new passage at a sentence boundary halfway the previous passage. For Wikipedia articles that contain fewer than 500 characters in total, we included the complete text as one passage. Our segmentation process produced an index of 6,365,890 passages. We separately saved the document title and section heading as metadata for each passage because they were used in our feature set.

For our question set, we exploited the Webclopedia question set by Hovy et al. (2002). This set contains questions that were asked to the online QA system answers.com. Of these questions, 805 (5\% of the total set) are why-questions. For development and testing

\footnotetext{
7 We assume that answer passages ending in an unfinished sentence are undesirable. However, if the hard maximum of 800 characters is reached, the passage is cut off between two words to prevent non-sentence contexts like tables to result in extremely long passages.
} 
purposes, we needed a set of questions for which we knew that they had an answer in the corpus. For 700 randomly selected why-questions from this set we therefore searched for an answer in the Wikipedia XML corpus by manually formulating queries and browsing through documents. Three examples illustrate the type of data we are working with:

1. "Why do most cereals crackle when you add milk?"- "They are made of a sugary rice mixture which is shaped into the form of rice kernels and toasted. These kernels bubble and rise in a manner which forms very thin walls. When the cereal is exposed to milk or juices, these walls tend to collapse suddenly, creating the famous 'Snap, crackle and pop' sounds."

2. "Why didn't Socrates leave Athens after he was convicted?"- "Socrates considered it hypocrisy to escape the prison: he had knowingly agreed to live under the city's laws, and this meant the possibility of being judged guilty of crimes by a large jury."

3. "Why was cobalt named cobalt?"- "The word cobalt comes from the German kobalt or kobold, meaning evil spirit, the metal being so called by miners, because it was poisonous and troublesome (it polluted and degraded the other mined elements, like nickel)."

For 186 of the 700 why-questions, we were able to find at least one correct the answer in the Wikipedia corpus. ${ }^{8}$ Thus, our data collection consists of 186 why-questions. This is not very large for machine learning experiments but comparable to the data collections that are contained in the LETOR benchmark data set (Qin et al. 2008).

\subsection{System set-up}

Our system consists of three modules that are run in sequence:

1. A question processing module that transforms the input question to a query by removing stop words and punctuation.

2. An off-the-shelf retrieval module that retrieves passages from the Wikipedia passage database that share content with the input query. Here, we use Lemur to retrieve $150^{9}$ answer passages per question. The selection of these 150 passages was done on the basis of the TF-IDF weighting scheme as it has been built in in Lemur (Zhai 2001). This gives us a set of 186 questions with 150 candidate answers per question.

3. A ranking module that ranks the retrieved passages using features extracted from the question and each of the 150 candidate answers (see Sect. 3.4 below): 27,900 $(186 * 150)$ question-answer pairs (instances) in total. One of the features that we use is the TF-IDF score that was assigned to each candidate answer by Lemur.

\subsection{Ground truth labeling}

For training and testing machine learning techniques, each instance in the data has to be assigned a label. In IR research, these labels are relevance assessments. In the case of learning-to-rank experiments, researchers are often faced with large amounts of data that

\footnotetext{
8 Thus, about $25 \%$ of our questions have an answer in the Wikipedia corpus. For the majority of the other questions (except for some questions that seem to be jokes rather than a serious information need), the coverage of Wikipedia in 2006 appeared not to be sufficient. A detailed analysis of Wikipedia's shortcomings for our data is not in the scope of this paper.

9 We experimented with a higher number of answer candidates but coverage was hardly improved when increasing this number to 500 .
} 
need to be labeled: a relatively small set of 100 queries with 100 results per query already gives a set of 10,000 instances. Since it is often too costly to label all instances by hand, estimations of relevance are generally used instead of complete manual judgments. These estimations can come from the aggregation of rankings by multiple systems for the same data, or by cleverly sampling a number of instances per cluster to find some that can be annotated as relevant; in this case all unannotated instances are considered irrelevant. A number of aggregation and sampling options for the estimation of relevance assessments are discussed in Aslam et al. (2009).

Thus, the large amounts of training instances force IR researchers to use estimations instead of complete manual judgments for labeling the instances. Moreover, in the case of why-QA, it is not possible to apply fully automatic ground truth labeling (which is often applied for evaluation in factoid-QA) because the answer to a why-question can have several textual variants. Therefore, we performed a form of sampling in which an assessor judged answers for each question, starting with the answer that is ranked first and stopping at the first correct answer found. We did this for several system settings, which gave different rankings and therefore different samples of assessments.

Although it is possible to judge the quality of answers to why-questions on a multi-level scale (ranging from partly relevant to highly relevant), we found that multi-level judgments are very subjective. Therefore, we decided to use binary relevance assessments: "Does this passage answer the question, or not?" Judgments by a second assessor on a sample of the data showed that the annotation task was relatively difficult: the two assessors agreed in $97 \%$ of the cases, but taking into account the chance agreement we reached only a moderate $\kappa$ value of 0.48 (due to the highly imbalanced data). Since the second assessor only judged a sample of the data that had been annotated by the first assessor, it was not sensible to try and reach consensus on these data with two annotators. Therefore, we used the ground truth annotations of the first assessor.

Because we judged a sample of all instances, we supported our manual judgments with a set of answer patterns similar to the answer patterns used in TREC: a regular expression for each question that defines which answers should be labeled as correct. With these answer patterns, we automatically labelled the unannotated instances: the answers that matched the answer pattern were labelled 'correct', the others 'incorrect' (instead of considering all unlabeled instances as incorrect). For example, for question 2 above ("Why didn't Socrates leave Athens after he was convicted?"), we developed the following answer pattern after assessing a sample of the candidate answers in our set: /(Socrates. * opportunity. $*$ escape.* Athens. * considered.* hypocrisy | leave. * run.* away.* community. * reputation)/. The pattern is based on two variants of the correct answer that we found in the set of candidate answers. ${ }^{10}$

\subsection{Feature extraction}

From earlier work (Verberne et al. 2010), we compiled a set of 37 features that are summarized in Table 1 . We syntactically parsed the questions with the Pelican parser ${ }^{11}$ and the candidate answers with the Charniak parser (Charniak 2000). Then we used a Perl script to extract all feature values from the question, the answer candidate and both their parse trees.

\footnotetext{
${ }^{10}$ Note that the vertical bar separates the two alternative formulations.

${ }^{11}$ See http://www.lands.let.ru.nl/projects/pelican.
} 
Table 1 Set of 37 features used in our ranking module

\begin{tabular}{lc}
\hline TF-IDF & $\begin{array}{c}\text { The score that is assigned to a candidate answer by Lemur/TF-IDF in the retrieval } \\
\text { module } \\
\text { Overlap between question and answer constituents (e.g. subject, verb, question } \\
\text { focus) } \\
14 \text { Syntactic features }\end{array}$ \\
$\begin{array}{c}14 \text { WordNet expansion } \\
\text { Overlap between the WordNet synsets of question and answer constituents }\end{array}$ \\
$\begin{array}{c}1 \text { Cue phrase feature } \\
\text { Overlap between candidate answer and a pre-defined set of explanatory cue } \\
\text { phrases }\end{array}$ \\
$\begin{array}{c}\text { Ovecument structure } \\
\text { features }\end{array}$ \\
$\begin{array}{c}\text { OverdNet Relatedness between question (focus) words and document title and section heading } \\
\text { feature }\end{array}$ & $\begin{array}{c}\text { Relatedness between question and answer according to the WordNet similarity } \\
\text { tool (Pedersen et al. 2004) }\end{array}$ \\
\hline
\end{tabular}

Each feature represents the overlap between two bags of item tokens ${ }^{12}$ : a bag of question item tokens (for example: all question's noun phrases, or the question's main verb) and a bag of answer item tokens (for example: all noun phrases in the answer, or all main verbs in the answer). The value that is assigned to a feature is a function of the overlap between these two bags. We used the following overlap function:

$$
S(Q, A)=\frac{Q_{A}+A_{Q}}{Q+A}
$$

in which $Q_{A}$ is the number of question item tokens that occur in the bag of answer item tokens, $A_{Q}$ is the number of answer item tokens that occur in the bag of question item tokens, and $Q+A$ is the number of item tokens in both bags of items joined together. So for instance, for the noun phrase overlap, $Q_{A}$ is the number of noun phrases in the question that also occur in the answer. $A_{Q}$ is then the number of noun phrases in the answer that also occur in the question, and $Q+A$ is the total number of occurrences of any noun phrase in the question and any noun phrase in the answer. The result is a value between 0 (none of the noun phrases in the question and the answer overlap) and 1 (all noun phrases in the question are present in the answer and the other way around).

\subsubsection{Description of the features}

Below we give a summary description of the 37 features that we used for ranking (cf. Table 1).

- Syntactic features. These are features that describe the overlap between a syntactically defined question part (such as subject, verb or direct object) and the answer passage or parts of the answer passage (e.g. matching the question's verb to all verbs in the answer). The syntactic features that deserve some extra attention here, are the features related to question focus (e.g. overlap between the question focus and the title of the answer document). In Verberne et al. (2010), we introduced the term question focus in analogy to linguistically motivated approaches to factoid QA for the topic of the question ("What is the question about?"). We defined three rules for determining the

\footnotetext{
12 Note that a 'bag' consists of individual tokens, while a 'set' consists of types. This makes our overlap measure different from the traditional overlap measures that calculate the overlap between sets (e.g. the Jaccard index).
} 
focus of a why-question: If the subject is semantically poor (people, human or a pronoun, $15 \%$ of why-questions), the question focus is the (verbal or nominal) predicate: "Why do peoplesneeze?". In case of etymology questions (10\% of whyquestions), the focus is the subject complement of the passive sentence: "Why are chicken wings calledBuffalo wings?". In all other cases, the focus is the syntactic subject of the question, e.g. "Why areflamingos pink?". These three rules cover all general domain why-questions, the majority category (75\%) being the latter (Verberne et al. 2010).

- WordNet expansion features. For each of the syntactic overlap features, we included an additional feature that describes the overlap between the WordNet synonym set (Fellbaum 1998) of a syntactically defined question part and the answer. This allowed us to investigate the importance of WordNet expansions for specific parts of the question, instead of for all question words indistinguishably.

- Cue phrase feature. The cue phrase feature is the overlap between the bag of answer words and a fixed set of words that suggest some kind of explanation. We found the cue phrases in a way that is commonly used for finding answer templates: we queried the key answer words to the one most frequent why-question on the web ("blue sky rayleigh scattering" for "Why is the sky blue?") to MSN's Live Search ${ }^{13}$ and crawled the first 250 answer fragments that are retrieved by the engine. From these, we manually extracted all phrases that introduce the explanation. This led to 47 cue phrases such as because, as a result of, which explains why, etc.

- Document structure features. The six document structure features cover information about the document context of a candidate answer passage, such as: the overlap between the question and the title of the Wikipedia document, the overlap between the question and the title of the section in which the candidate answer occurs, and the relative position of the candidate answer in the document.

- WordNet Relatedness feature. We defined the relatedness between a question and an answer as the average of the relatedness of each question word with all words in the answer:

$$
\operatorname{REL}(Q, A)=\frac{\sum_{q=1}^{m} \sum_{a=1}^{n} \operatorname{REL}\left(w_{q}, w_{a}\right)}{m}
$$

in which $Q, A$ is the question-answer pair under consideration, $w_{q}$ represents the question words, $w_{a}$ the answer words, $m$ is the number of question words, and $n$ is the number of answer words. As a measure of word relatedness $\left(\operatorname{REL}\left(w_{q}, w_{a}\right)\right)$, we chose the Lesk measure, which finds overlaps between the glosses of two words, also if they belong to different word classes (Pedersen et al. 2004). We used the version of Lesk that was adapted for WordNet by Banerjee and Pedersen (2002).

\subsubsection{Resulting feature vectors and normalization}

Feature extraction led to a vector comprising 37 feature values for each of the 27,900 items in the data set. For feature value normalization, we performed a form of clusterwise normalization that is comparable to the approach by Liu et al. (2007) ('QueryLevelNorm' in LETOR).

13 See http://www.live.com. 
Assume a question $Q_{i}$ with the candidate answers $A_{j}(j=1 \ldots 150)$. For each feature $F_{k}$ ( $k=1 \ldots 37)$, its value $x_{i j k}$ is normalized by transforming it to its $z$-score:

$$
x_{i j k}^{\prime}=\left(x_{i j k}-\mu_{i k}\right) / \sigma_{i k}
$$

in which $\mu_{i k}$ is the mean of all values of feature $F_{k}$ for the candidate answers to $Q_{i}$ and $\sigma_{i k}$ is the standard deviation of all values of feature $F_{k}$ for the candidate answers to $Q_{i}$.

Normalizing feature values to a relative value within a cluster makes our data more suitable for pointwise learning approaches. Moreover, this approach makes it possible to normalize the scores independently of the answers to other questions: It can be performed for the set of candidate answers to each new input question.

\subsection{Evaluation set-up}

Each instance in our data was labeled correct if the candidate answer was deemed a correct answer to the question and incorrect if it was not (see Sect. 3.3). On average, a whyquestion had 1.6 correct answers among the set of 150 candidate answers retrieved by Lemur. This means that the incorrect/correct ratio in our data collection is 71 to $1(98.6 \%$ of the instances in the training set was labeled incorrect). Akbani et al. (2004) consider a data set to be 'highly imbalanced' for the use of classification techniques if the ratio of negative against positive instances is bigger than 50 to 1 .

For evaluation, we counted the questions in the test set that have at least one correct answer in the top $n(n \in 10,150)$ of the results. This number divided by the total number of questions in our test collection gave the measure Success@n. For the highest ranked correct answer per question, we determined its reciprocal rank $(\mathrm{RR}=1 / \mathrm{rank})$. If there was no correct answer retrieved by the system at $n=150$, the RR was 0 . Over all questions, we calculated the mean RR (MRR). ${ }^{14}$

We performed fivefold cross validation on the question set. We kept the 150 answers to each question together in onefold so that we did not train and test on answers to the same question. For techniques that require tuning of hyperparameters, we used a development set (see Sect. 4.1). In the training stage, we excluded the 40 questions (21.5\%) for which none of the 150 candidate answers was correct. The test set on the other hand did contain these questions, for which RR would naturally be 0 .

\section{Experiments}

In this section, we describe the machine learning techniques we evaluated and how we applied each of them to our learning problem. In all cases, we used the 37 -feature set with clusterwise normalization that we described in Sect. 3.4. As a baseline, we used the system setting in which the answers are retrieved and ranked according to TF-IDF only.

An overview of our data collection, together with the set-up of evaluation and hyperparameter tuning, can be found in Table 2 .

\footnotetext{
${ }^{14}$ MRR is a useful measure for query types that have only one correct answer. For factoid questions, for example, retrieving multiple occurrences (or variants) of the same answer does not provide the user with useful new information, so only the highest ranked correct answer is counted in the evaluation. As we saw in our data set, why-questions have one or very few correct answers, making MRR a suitable evaluation measure.
} 
Table 2 Overview of our data collection, and the set-up of evaluation and hyperparameter tuning

\begin{tabular}{ll}
\hline Demographics of the data & \\
Number of questions (and consequently answer clusters) in data & 186 \\
Number of candidate answers per question & 150 \\
Total number of instances & 27,900 \\
Number of features & 37 \\
Percentage of instances labeled as incorrect & $98.6 \%$ \\
Evaluation set-up and hyperparameter tuning & 5 \\
Number of folds used in cross-validation & 37 \\
Mean number of questions per test set (onefold) & 117 \\
Mean number of questions per training set (fourfolds; successful questions only) & 10 \\
Number of questions per development set (held out of training data) & 3 \\
Number of development sets per training set & \\
\hline
\end{tabular}

\subsection{Matrix of techniques}

We compared the three learning-to-rank approaches introduced in Sect. 2.2: the pointwise approach (see Sect. 4.2), the pairwise approach (Sect. 4.3) and the listwise approach (Sect. 4.4). In the pointwise approach, we evaluated the following classification and regression techniques: Naïve Bayes, Support Vector Classification, Support Vector Regression and Logistic Regression. In the pairwise approach, we evaluated the same classification and regression techniques, and Ranking SVM. For the listwise approach, we evaluated $S V M^{\text {map }}$ and a Genetic Algorithm that optimizes for MRR.

\subsubsection{Hyperparameter tuning}

For techniques that require hyperparameter values, we not only evaluated the default hyperparameter setting but we also tried to find optimal values for the hyperparameters using a grid search over a large range of values (see Sect. 4.2 for a description of the grid we used). For hyperparameter tuning, it is necessary to use development data that is held out from the training set. We searched for hyperparameter values that give the best results in terms of MRR on the development set. Given the small number of questions in our training set, ${ }^{15}$ we decided to hold out 10 questions with their 150 answers from each training set. Because development sets of 10 questions are quite small, we selected three (non-overlapping) development sets for each fold.

As a further measure to prevent overfitting on the development sets (in order to make the optimization process more robust), we selected three (near-)optimal hyperparameter settings for each development set, instead of simply taking the one leading to the best MRR. The three hyperparameter settings were selected as follows: The first was always the one leading to the best MRR on the development set. The second and third were the highest local optima that are further than five steps in the grid away from the first chosen point and from each other (see the descriptions of the used grids in 2).

15 Around 117 because, as explained in Sect. 3.5, we excluded the $21 \%$ questions without correct answers and $20 \%$ for each fold to test on. 
During testing, the outputs of the nine models that were created for the three development sets (three models per development set) were combined by addition, after scaling them to a comparable range.

\subsection{The pointwise approach}

We first investigated the pointwise approach of applying classification and regression techniques to our learning problem. In the training phase, the classifier or regressor learns to classify each instance (question answer pair) as either correct or incorrect, irrespective of the cluster it belongs to. ${ }^{16}$ In the test phase, we let the model assign a score to each instance in the data representing the likelihood that this instance should be classified as correct. The actual ranking is done by a script that sorts the instances per cluster by the output score of the classifier.

As discussed in Sect. 3.5, our data show a strong imbalance between positive and negative instances, with a incorrect/correct ratio of 71 . This may cause problems for machine learning techniques that are designed for classification. Therefore, we applied a balancing strategy to all classification and regression techniques that we evaluated. As observed in the literature (Japkowicz and Stephen 2002; Tang et al. 2009), applying a cost factor is the preferred approach to counter imbalance. If a system did not allow for this, we applied oversampling of the positive instances in such a way that each training set included approximately as many positive as negative instances.

In the pointwise approach, we trained and tested each machine learning technique both on the original (imbalanced) data and on the data that was balanced first (by applying a cost factor or oversampling). For the classifiers that allow for hyperparameter optimization, we performed the optimization for both these data variants. This led to two (when hyperparameter optimization is not feasible) or four different settings per machine learning technique: original default, original tuned, balanced default, and balanced tuned.

\subsubsection{Naïve Bayes classifier (NB)}

For experiments with Naïve Bayes (NB), we used the e1071 package in $R .^{17}$ This package does not allow for tuning of hyperparameters for Naïve Bayes so we only ran the Naïve Bayes classifier in its default setting, on both the original and the oversampled data.

\subsubsection{Support vector classification and support vector regression (SVR)}

For standard support vector methods, we used LIBSVM. ${ }^{18}$ As proposed by the authors of LIBSVM, we first scaled our data using svm-scale. We experimented with support vector classification (C-SVC) and support vector regression ( $\varepsilon$-SVR). For both, we used the RBF kernel (Hsu et al. 2003).

The RBF kernel expects two hyperparameters: $c$ - the trade-off between training error and margin, and $\gamma$-a multiplication factor determining the range of kernel space vector norms. Their default values are $c=1$ and $\gamma=1 / k$ with $k$ being the number of features, giving a $\gamma$ of 0.027 for our data. For the grid search, we followed the suggestion in Hsu

\footnotetext{
${ }^{16}$ Recall that we did normalize the feature values per cluster, which made our data more suitable for pointwise learning approaches.

17 See http://www.cran.r-project.org/web/packages/e1071/index.html.

18 See http://www.csie.ntu.edu.tw/ cjlin/libsvm.
} 
et al. (2003) to use exponentially growing sequences of $c$ and $\gamma$. We varied $c$ from $2^{-13}$ to $2^{13}$ in steps of $\times 2$ and $\gamma$ from $2^{-13}$ to $2^{7}$ in steps of $\times 4 .^{19}$

Support vector classification allows us to use a cost factor for training errors on positive instances, which we did: During hyperparameter tuning, we kept the cost factor unchanged at $71(-w 1=71)$. For SVR (which does not allow for a cost factor), we oversampled the positive instances in the training sets.

\subsubsection{Logistic regression (LRM)}

We used the $1 \mathrm{rm}$ function from the Design package in $R$ for training and evaluating models based on logistic regression. ${ }^{20}$ LRM uses Maximum Likelihood Estimation (MLE) as optimization function. We used 'correct' and 'incorrect' as target values in the training phase. In the test phase, the regression function is applied to the instances in the test set, predicting for each item the log odds that it should be categorized as 'correct'.

LRM has a built-in option for data balancing (applying a weight vector to all instances), of which we found that it has exactly the same effect on the data as oversampling the positive instances in the training set. The other hyperparameters in LRM (a parameter for handling collinearity in stepwise approaches ${ }^{21}$ and a penalty parameter for data with many features and relatively few instances) are not relevant for our data. Therefore, we refrained from hyperparameter tuning for LRM: We only trained models using the default parameter settings for both the original and the balanced data.

\subsection{The pairwise approach}

For the pairwise approach, we evaluated Joachim's algorithm Ranking SVM (Joachims 2002). In addition, we evaluated the same classification and regression techniques as in the pointwise approach. We made this possible by transforming our data into instance pairs that can be handled by these techniques (as explained below).

\subsubsection{Ranking SVM}

We used version 6 of $S V M^{\text {light }}$ for our Ranking SVM experiments. ${ }^{22}$ Ranking SVM considers the training data to be a set of instance pairs, each pair consisting of one correct and one incorrect answer. On these instances, the system performs pairwise preference learning (Joachims 2002). The ranking order of a set of training instances is optimized according to Kendall Tau:

$$
\tau=\frac{\left(N_{\mathrm{c}}-N_{\mathrm{d}}\right)}{\left(N_{\mathrm{c}}+N_{\mathrm{d}}\right)}
$$

in which $N_{\mathrm{c}}$ is the number of concordant item pairs (the two items are ordered correctly) and $N_{\mathrm{d}}$ is the number of discordant item pairs (the two items are ordered incorrectly).

\footnotetext{
19 This means that each next value is 4 times as high as the previous, so we go from $2^{-13}$ to $2^{-11}$ to $2^{-9}$, etc.

20 See http://www.cran.r-project.org/web/packages/Design/index.html.

21 We only built regression models that contained all features as predictors.

22 See http://www.svmlight.joachims.org.
} 
Similar to the other SVM techniques, we used the RBF kernel in Ranking SVM, which takes both the hyperparameters $c$ and $\gamma$. For both, the default value is 1 . For tuning these parameters, we searched over the same grid as for SVC.

\subsubsection{Classification and regression techniques}

To enable the use of other classifiers than Ranking SVM and regression techniques in a pairwise approach, we transformed our data into a set of instance pairs, similarly to the approach implemented by Joachims in Ranking SVM. We presented the answers in pairs of one correct and one incorrect answer to the same question. We kept the number of features constant (at 37), but we transformed each feature value to the difference between the values of the two answers in the pair. In other words, we created feature vectors consisting of 37 difference values.

In the training data, each instance pair is included twice: 'correct minus incorrect' with label 'correctly ordered' and 'incorrect minus correct' with label 'incorrectly ordered'. In the testing phase, we let the classifier assign to each instance pair the probability that it is correctly ordered. Then we transform the data back to normal answer instances by summing the scores for each answer $i$ over all pairs $[i, j]$ in which $i$ is ranked first.

We evaluated the same classifiers and regression techniques in the pairwise approach as we evaluated for the pointwise approach: Naïve Bayes, Support Vector Classification, Support Vector Regression and Logistic Regression. Although our implementation of pairwise classification for SVC is conceptually equal to Ranking SVM, there are some implementational differences (such as the use of LIBSVM vs. $S V M^{l i g h t}$ ). ${ }^{23}$ We decided to evaluate both so that we could maintain a link to previous work as well as get more direct comparability with the other pairwise classification and regression methods.

\subsection{The listwise approach}

In the listwise approach there is no classification of instances or instance pairs; instead, the ordering of an answer cluster as a whole is optimized. As explained in Sect. 2.2, the implementation of listwise ranking approaches is a recent development and the results obtained with these techniques are promising (Yue et al. 2007; Xu and Li 2007). We evaluated two listwise optimization algorithms: $S V M^{\text {map }}$, and our own implementation of a genetic algorithm that optimizes the order of answers per question using MRR as fitness function. $^{24}$

\subsubsection{SVM map}

$S V M^{\text {map }}$ is a freely available algorithm ${ }^{25}$ that takes clusters of instances with binary relevance labels as input and optimizes the instance order within each cluster for Mean Average Precision (MAP) (Yue et al. 2007). Average precision is calculated as:

\footnotetext{
${ }^{23}$ The default value for $\gamma$ is $1 / k$ in LIBSVM and 1 in $S V M^{\text {light }}$.

24 To our knowledge, there are no implementations of AdaRank and ListNet available for download and to experiment with.

25 See http://www.projects.yisongyue.com/svmmap.
} 


$$
\mathrm{AP}=\frac{\sum_{j: P_{j}=1} \text { Prec@j }}{\text { rel }},
$$

in which $j: P_{j}=1$ represents each position in the result list where a relevant result is found, rel is the number of relevant documents and Prec@j is the precision at position $j$. MAP is the mean AP over all queries in the data.

In $S V M^{\text {map }}$, we again used the RBF kernel. For tuning the parameters $c$ and $\gamma$, we searched over the same grid as for SVC.

\subsubsection{Genetic algorithm (GA)}

We used a Perl implementation of a genetic algorithm (GA) (Goldberg et al. 1988) for our experiments. ${ }^{26}$ Our aim when training the genetic algorithm was to find the optimal weight vector for our feature vector of 37 feature values (a linear combination of feature values). As weights, we used the integers $0-10$. In terms of the genetic algorithm, each possible weight vector is an individual.

In each run ('generation'), the GA selects the configurations that give the highest MRR on the training set (the 'fittest individuals') for crossover ('mating'). By default, the crossover rate is 0.95 and the mutation rate 0.05 . For the selection of individuals, we chose tournament selection, which is the most efficient strategy. We used uniform crossover because the order of our features in the feature vector is not relevant. In our experiments, we set the generation size to 500 and the number of generations to 50 based on the shape of the learning curve in earlier experiments on the same data.

We did not run a meta-level GA for tuning our GA, because implementing such a procedure proved to be computationally prohibitive.

\section{Results}

The results that we obtained in terms of MRR are in Table 3. In addition to MRR, we calculated success@150 and success@10 (not shown in Table 3). For all settings, success@150 is $78.5 \%$ (This score does not change because there are no new answers retrieved by the ranking module). Success@10 is around 56\% for the best-scoring settings (compared to $45 \%$ for the TF-IDF baseline). For some of the techniques, we also calculated MAP. As expected, the figures for MAP closely resemble the results in terms of MRR because the data contain only a small number of correct answers.

In addition to a comparison between different techniques, we present detailed results for LRM in Table 4. We chose LRM for this because the MRR score is not significantly lower than the optimal MRR, and it allows us to see which features made a significant contribution to the ranking model and the coefficients that were assigned to them. For a more detailed discussion of the significant features we refer to Verberne et al. (2010).

For significance testing, we used the Wilcoxon Signed-Rank test on paired reciprocal ranks (RRs): Per question, we took the RR of the highest ranked correct answer in two system settings. Then we made 186 pairs of RRs for these two settings and calculated the Wilcoxon score over them.

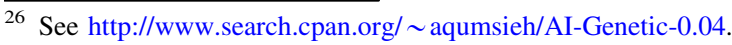


Table 3 Results for the pointwise, pairwise and listwise approaches in terms of MRR

\begin{tabular}{lllll}
\hline Technique & \multicolumn{2}{l}{ Pointwise approach } & & \\
\cline { 2 - 5 } & Original default & Original tuned & Balanced default & Balanced tuned \\
\hline TF-IDF & 0.25 & & & - \\
NB & 0.19 & - & 0.20 & $0.33^{*, \dagger}$ \\
SVC & 0.10 & $0.32^{*, \dagger}$ & $0.32^{*, \dagger}$ & $0.32^{*, \dagger}$ \\
SVR & $0.34^{*, \dagger}$ & $0.30^{*}$ & $0.33^{*}$, & - \\
LRM & $0.34^{*, \dagger}$ & - & $0.31^{*}$ & - \\
\hline
\end{tabular}

\begin{tabular}{llr}
\hline Technique & Pairwise approach & Tuned \\
\cline { 2 - 3 } & Default & - \\
\hline NB & $0.32^{*, \dagger}$ & $0.34^{*}$, \\
SVC & $0.32^{*, \dagger}$ & $\mathbf{0 . 3 5}^{*}$ \\
SVR & $0.32^{*, \dagger}$ & - \\
LRM & $0.31^{*}$ & $0.33^{*}, \dagger$ \\
Ranking SVM & 0.13 & Tuned \\
\hline Technique & Listwise approach & - \\
\cline { 2 - 3 } & Default & $0.34^{*}, \dagger$ \\
\hline GA-MRR & $0.32^{*, \dagger}$ & $0.33^{*, \dagger}$ \\
SVMap & & \\
\hline
\end{tabular}

The highest score is printed in boldface. An asterisk (*) on an MRR score indicates a statistically significant improvement $(P<0.01$ according to the Wilcoxon signed-rank test) over the TF-IDF baseline. A dagger $(\dagger)$ indicates that the MRR score is not significantly lower than the highest MRR score (0.35)

Table 4 Detailed results for LRM on original data in terms of MRR, MAP, Success@ 10, Success@ 150, and the coefficients of the features that significantly contribute to the re-ranking score $(P<0.05)$, ranked by their coefficient in LRM (representing their strength)

\begin{tabular}{lr}
\hline Mean reciprocal rank (MRR) & 0.34 \\
Mean average precision (MAP) & 0.32 \\
Success@10 & $57.0 \%$ \\
Success@150 & $78.5 \%$
\end{tabular}

Significant features

Coefficient

TF-IDF

Overlap between question focus synonyms and document title $\quad 0.25^{* *}$

$\begin{array}{ll}\text { Overlap between question object synonyms and answer words } & 0.22\end{array}$

Overlap between question object and answer objects $0.18^{*}$

$\begin{array}{lc}\text { Overlap between question words and document title synonyms } & 0.17\end{array}$

Overlap between question verb synonyms and answer words 0.16

WordNet Relatedness $\quad 0.16^{*}$

$\begin{array}{ll}\text { Cue phrases } & 0.15^{*}\end{array}$

Asterisks on coefficients denote the level of significance for the feature: ** means $P<0.001$, * means $0.001<P<0.01$, no asterisk means $0.01<P<0.05$ 
The highest MRR score that we obtained is 0.35 (by SVR for pairwise classification). ${ }^{27}$ We will call this the optimum in the remainder of this section.

\subsection{Comparing pointwise, pairwise and listwise approaches}

We obtained good results with techniques following either of the three approaches: pointwise, pairwise and listwise. The results for the pairwise approach much resemble the results for balanced data in the pointwise approach. This finding confirms the results found by other researchers on LETOR data: pairwise approaches are in some cases slightly better than pointwise approaches but pointwise approaches can reach good results if the data are balanced and the hyperparameters are tuned properly.

For Naïve Bayes, however, the results for the pointwise and pairwise approaches are very different. Here we see that presenting the problem as a pairwise classification problem is essential for Naïve Bayes to predict the data correctly. We suspect that this is because the simplicity of the Naïve Bayes model, which is based on the probability of each feature value given the class of the instance. When presenting the data in pairs, we apply a form of bagging: Each positive answer is included in the data many times, but each time as part of a different instance pair. As a result, all positive instance pairs are different from each other and the algorithm has more more quasi-independent data points available for learning to make the right decision for one answer. Not surprisingly, the Naïve Bayes classifier depends on the availability of (quasi-)independent training data for learning a proper classifier.

In the bottom part of Table 3, we see that both our listwise approaches (GA-MRR and $S V M^{\text {map }}$ ) lead to scores that are not significantly lower than the optimum, but also not higher than the results for the pointwise and pairwise techniques. From the literature on listwise techniques one would expect a result that is better than the pointwise and pairwise approaches. We speculate that the failure of our GA approach to outperform pointwise and pairwise approaches is because the linear feature combination with integer weights that we implemented in the Genetic Algorithm is not sophisticated enough for learning the data properly. The results for $S V M^{\text {map }}$ may be suboptimal because the optimization is done on a different function (MAP) than the evaluation measure (MRR). We [and others (Liu 2009)] have found before that the best results with listwise techniques are obtained with a loss function that optimally resembles the evaluation measure. In that respect, it would be interesting to experiment with the lesser known algorithm $S V M^{m r r}$ (Chakrabarti et al. 2008).

\subsection{The effect of data imbalance}

As pointed out in the machine learning literature (see Sect. 2.3), classifiers are in general sensitive to data imbalance. Table 3 shows that especially pointwise SVC gives very poor results in its default setting on our imbalanced data. If we balance the data, SVC reaches good results with the default settings of LIBSVM. As opposed to SVC, the results for Naïve Bayes are not improved by balancing the data.

\footnotetext{
27 The $21 \%$ of questions without a correct answer in the top 150 all have an RR of 0; the MRR for the successful questions only is 0.45 . This is quite high considering the Success@10 score of 56\%. A further investigation of the results shows us that this is because a large proportion of successful questions has a correct answer at position 1 (Success@1 for all questions including the unsuccessful questions is 24.2\%).
} 
We find that for regression techniques (SVR and LRM), balancing the data by oversampling or applying a cost factor leads to slightly (not significantly) lower MRR scores. In Sect. 6.2 we provide an analysis of the regression models for the original and balanced data.

\subsection{The effect of hyperparameter tuning}

The effects of hyperparameter tuning on the ability of a technique to model our data varies much between the different techniques. The results for pointwise SVC show that with optimal hyperparameter settings SVC is able to reach a good result, even for the highly imbalanced data, on which the default settings performed very poorly. Ranking SVM, for which the default settings also give a result below baseline, also profits significantly from hyperparameter optimization $(P<.0001)$.

On the other hand, for those settings where default hyperparameters already give good results (most pointwise approaches on the balanced data and most pairwise approaches), we see that hyperparameter tuning does not lead to significantly better MRR scores. The only exception is pairwise SVR with $P=0.01$ according to Wilcoxon.

There is one setting where we even observe a significant $(P=0.0003)$ lower MRR score after hyperparameter tuning compared to the default setting: pointwise SVR on the original data. In Sect. 6.3, we will provide an analysis of the tuning process in order to see if our experiments suffer from overfitting. Additionally, we will analyze the differences between individual questions in Sect. 6.4.

\section{Discussion}

\subsection{Relating our findings to previous work}

Although our feature set is different from the feature sets that are used in previous work on learning to rank for Information Retrieval, there are also similarities between our data and more commonly used data in learning-to-rank research: in all data, the instances are clustered by query, and there is often a strong imbalance between positive and negative instances.

The performance differences that we find between pointwise, pairwise and listwise approaches are small, especially after balancing the data. We argue that this is largely in line with earlier findings in learning-to-rank for Information Retrieval, where differences between the three approaches on smaller datasets are not always significant (Liu 2009).

We can identify four distinct reasons why some of our findings are different from the findings in the learning-to-rank literature. First of all, our data collection is relatively small (186 questions), as a result of which it is difficult to get differences that are statistically significant. The same pattern of listwise methods being not significantly better than pairwise and pointwise methods can be observed for some of the smaller LETOR collections such as OHSUMED, which contains only 106 queries (Liu et al. 2007).

Secondly, we performed clusterwise normalization on the data for all techniques. As a result, the feature values of one candidate answer in the training set are related to the feature values of other candidate answers to the same question. This gives pointwise techniques 'awareness' of the clustering of the answers per question. Thirdly, in hyperparameter tuning, we optimized for MRR on the development set. This means that all techniques profit from the advantage of optimizing for the measure that is used for 
evaluation as well. Finally, the listwise techniques that we evaluated are suboptimal: the genetic algorithm only performs a linear combination of feature values using integer weights; $S V M^{\text {map }}$ optimizes for a different measure than our main evaluation measure.

\subsection{Analyzing the effect of data imbalance}

In Sect. 5.2, we found that for regression techniques, balancing the data by oversampling or applying a cost factor leads to slightly (not significantly) lower MRR scores. In Sect. 2.3, we concluded from the literature that class imbalance causes fewer problems for regression techniques than for classifiers because in the regression model, the intercept value moves the outcome of the regression function towards the bias in the data.

An analysis of the regression models that were created by LRM for the original and the balanced data showed that the model for the balanced data contains much more significant effects (features that significantly contribute to the ranking model) than the model for the original data. Due to oversampling the positive instances in the training set, the regression function was strongly influenced by the heavy data points that each represent 71 positive instances. Misclassifying some of the negative instances during training does not influence the model fit too badly because there are much more positive data points. However, when applying the model to generate a ranking, the few negative instances that are misclassified are ranked above the positive instances. The MRR, which only considers the positive instance that is ranked highest, has therefore decreased.

\subsection{Analyzing the effect of hyperparameter tuning}

In Sect. 5.3, we reported that for those settings where default hyperparameters already give good results, hyperparameter tuning does not lead to better MRR scores. To examine whether overfitting on the development sets was the cause of this, we analyzed the results for one of the methods where a slight deterioration in MRR was observed between default and tuned hyperparameter settings: pointwise SVR on balanced data. For this setting, we plotted the measured MRR for every grid point for all tune and test runs. We then compared the surfaces for corresponding tune and test runs.

In the ideal situation, the surfaces would not need to coincide, but at least the highest peaks should be close to each other in the grid. Although this is the case for a few runs, the typical situation is that in Fig. 1, where the tune and test surfaces for fold 4, development set $\mathrm{A}$ are shown. The grid area where the peak occurs in the tune run has a lower than average MRR in the test run.

We also observe that the surfaces are not very smooth and sometimes show strong local jumps. This means that for a determination of the real optimal hyperparameters for each run, a denser grid would be preferable. However, seeing the mismatch between the surfaces for tune and test runs, we expect that this would not solve the problem of lower MRR scores after tuning. Thus, the process of hyperparameter tuning is hampered by the sparseness of our data.

The large differences between the optimal hyperparameter values that are found for different development sets shows that the optimal hyperparameter values are highly influenced by the individual questions in each development set. We conclude that for tuning purposes, a development set size of ten questions is clearly too small. Our strategy of using three development sets per training set has probably alleviated, but certainly not solved this problem. 

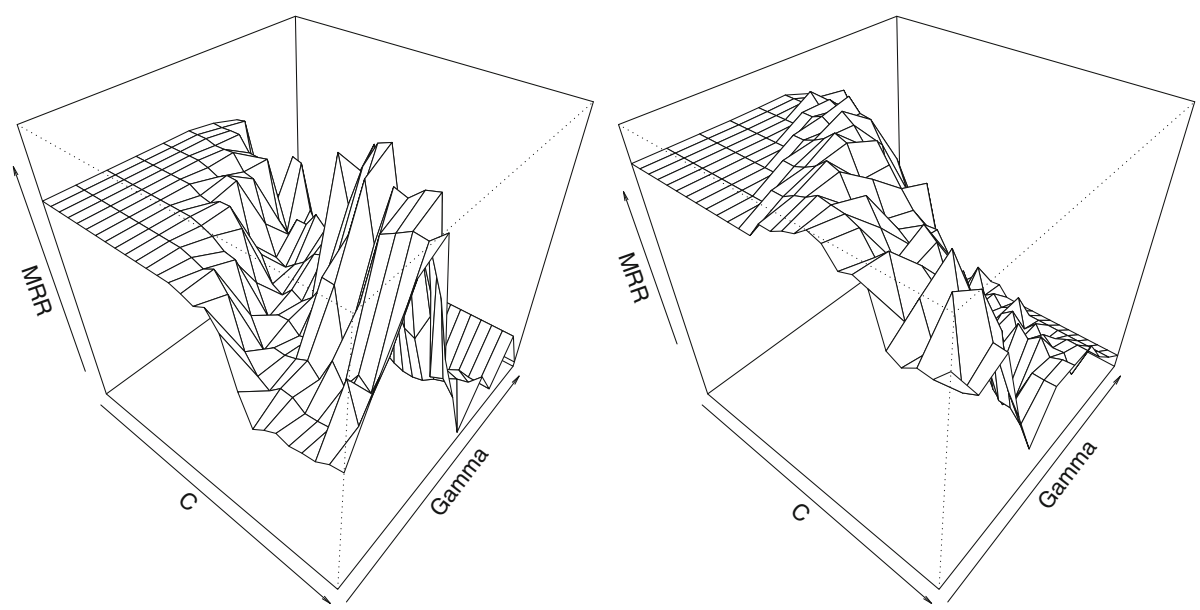

Fig. 1 The measured MRR for every grid point (parameters $\mathrm{c}$ and $\gamma$ ) for one tune run (left) and the corresponding test run (right), on the same grid

\subsection{Analyzing the effect of ranking on individual questions}

We analyze the effect of the learned ranking models on the level of individual questions. Per question, we compare the results that are obtained by the TF-IDF baseline, and each of the best performing 16 settings. We find that questions can be subdivided in five categories according to the success that the baseline and the experimental settings have on them. These categories are listed in Table 5, together with the percentage of questions that fall in each category and an example question.

Categories 1 and 6 are the least interesting categories, because these questions have a correct answer in the top-10 for either all or none of the settings (including the baseline). We could refer to the questions from category 1 as the 'easy' questions in our data, for which the TF-IDF baseline is already successful and additional ranking features do not harm the ranking. For the questions in category 6, there is no correct answer in the result list; consequently, no correct answer can be ranked in the top-10.

The questions in category 3 as the questions that are the most difficult to rank: there is a correct answer available in the result list but none of the settings is able to rank it into the

Table 5 All questions in our data collection subdivided in categories according to the success that the baseline and the 16 best experimental settings have on them. For each category, an example question is given

Questions answered in top-10 for... Example

All settings including baseline

Some settings excluding baseline

None of the settings

Some settings including baseline

TF-IDF baseline only

Questions with no correct answer
$37 \%$ Why are hush puppies called hush puppies?

$25 \%$ Why did the Globe Theatre burn down?

9\% Why did Greek philosophy begin in Miletus?

$7 \%$ Why are there no white lines on pro footballs?

$1 \%$ Why do men button from the right and women from the left?

$21 \%$ Why is English the language of the USA? 
top-10. Category 2 contains the questions where the learned ranking models are successful while the TF-IDF baseline is not; for most of these questions, all ranking models cause a correct answer to enter the top-10. These questions have a syntactic and semantic structure that optimally fits the important ranking features, such as the overlap between question focus and the title of the answer document in Wikipedia (e.g. the Globe Theatre). The relatively small category 4 contains the questions for which the ranking models have a mixed result: the TF-IDF baseline was able to rank a correct answer in the top-10 but some of the other ranking models are not. Unfortunately, the mixed results do no show a clear distinction between pointwise and pairwise techniques or techniques that work on original or balanced data.

The very small category 5 contains the two questions for which applying the additional ranking features causes the correct answer to drop out of the top-10. The features that are the most important for the data as a whole have a counterproductive effect on these questions (e.g. the subject men in the example question is bears very little semantic content as opposed to the verb button-this is a non-standard pattern in why-questions).

\subsection{Computational issues}

There is an additional factor in determining the best modeling method, apart from performance measures: the computational cost. We should distinguish here between the cost for training and the cost for actual application. The latter should not be of major importance. Assuming a starting point of 150 potential answers, application of a pointwise model, even with an RBF kernel, takes on average $0.8 \mathrm{~s}$ per question (average over all potential hyperparameters for pointwise SVR, oversampled). This time increases when using pairwise models that have a higher number of instances per question. Still, this should not present a problem in most practical applications.

More serious considerations come into play when examining the training process, especially for methods where hyperparameter tuning is needed. The optimal parameters for the model will have to be determined, often in an iterative process, for every possible hyperparameter grid point. For our dataset, pointwise SVR (oversampled) leads to a total training time of 18 days on a $2.33 \mathrm{GHz}$ Intel Core 2 Duo, which means an average training time per grid point of $350 \mathrm{~s}$, ranging from $4.75 \mathrm{~s}$ for $c=2048, \gamma=32$ up to $3.6 \mathrm{~h}$ for $c=8192, \gamma=0.5$. For a practical application, however, we would advise a much larger data set. This will inevitably increase the training time, although the increase might be partly offset by a quicker convergence in the optimalisation process due to a less sparse data set. On the other hand, the training obviously needs to be done only once, and added quality might make it worthwhile, despite the high training cost.

\section{Conclusion}

In this paper, we have optimized the re-ranking module of a system for why-question answering. The goal of this paper was to compare a number of machine learning techniques in their performance on the task of learning a ranking for answers that are described by TF-IDF and a set of 36 linguistically-motivated overlap features and a binary label representing their correctness. We evaluated learning techniques in pointwise, pairwise and listwise approaches. 
We found that with all machine learning techniques, we can get to an MRR score that is significantly above the TF-IDF baseline of 0.25 and not significantly lower than the best score of 0.35 .

We are able to obtain good results with all three types of approaches for our data: pointwise, pairwise and listwise. The optimum score was reached by Support Vector Regression for the pairwise representation, but some of the pointwise settings reached scores that were not significantly lower than this optimum. We argue that pointwise approaches can reach good results for learning-to-rank problems if (a) data imbalance is solved before training by applying a cost factor or oversampling the positive instances, (b) feature value normalization is applied per answer cluster (query-level normalization) and/ or (c) proper hyperparameter tuning is performed.

We obtained reasonable results with two listwise techniques: $S V M^{\text {map }}$ and a Genetic Algorithm (GA) optimizing for MRR. With these techniques, we obtained results that are comparable to those obtained with the pointwise and pairwise approaches. Given our suboptimal choice of optimization function $\left(S V M^{m a p}\right.$ vs. $\left.S V M^{m r r}\right)$ and our relatively simple implementation with a linear combination of feature values in the GA, we think that our results confirm the earlier findings that listwise approaches to learning-to-rank are promising.

We found that for our imbalanced data set, some of the techniques with hyperparameters heavily depend on tuning. However, if we solve the class imbalance by balancing our data or presenting the problem as a pairwise classification task then the default hyperparameter values are well applicable to the data and tuning is less important. The pairwise transformation enables even Naïve Bayes to classify and rank the data properly. Since hyperparameter tuning is a process that takes much time and computational power, a technique without hyperparameters, or a technique for which tuning can be done easily without heavy computing, should be preferred if it reaches equal performance to techniques with (more heavy) tuning. In this respect, regression techniques seem the best option for our learning problem: logistic regression reaches a score very close to the optimum (MRR is 0.34 ) without tuning. Pairwise support vector regression reaches optimal performance (MRR is 0.35) with tuning.

It seems that with the current feature set we have reached a ceiling as far as individual rankers are concerned. We might still achieve an improvement with a combination of rankers or second level classifiers, but the overlap between the results for individual systems is such that this improvement can never be very big. ${ }^{28}$

Moreover, given the experimental result of 0.35 and the theoretical optimum of 0.79 (if for all questions with at least one correct answer a correct answer is ranked at position 1), we can conclude that our features are suboptimal for distinguishing correct from incorrect answers. Since we already invested much time in previous work in finding the best features for describing our data, we conclude that the problem of distinguishing correct and incorrect answers to why-questions is more complex than an approach based on textual (overlap) features can solve.

Our future work will review the problem of answering why-questions in detail. In automatically answering complex questions such as why-questions, human reasoning and

\footnotetext{
${ }^{28}$ In fact, we did some preliminary experiments with a linear combination of answer scores from combinations of rankers. By use of a hill-climbing mechanism we were able to find a set of weights that leads to an MRR on the test set of 0.38 . This is significantly better than the best-scoring individual technique $(P=0.03)$. We have not attempted second level classification, since this would mean repeating all our experiments with a nested cross-validation in order to do a proper training and tuning of the second level classifier.
} 
world knowledge seem to play an important role. We will investigate the limitations of an Information Retrieval-based approach that relies on word overlap for complex question answering.

Open Access This article is distributed under the terms of the Creative Commons Attribution Noncommercial License which permits any noncommercial use, distribution, and reproduction in any medium, provided the original author(s) and source are credited.

\section{References}

Akbani, R., Kwek, S., \& Japkowicz, N. (2004). Applying support vector machines to imbalanced datasets. In Lecture notes in computer science: Machine learning: ECML 2004 (Vol. 3201, pp. 39-50). New York: Springer.

Aslam, J. A., Kanoulas, E., Pavlu, V., Savev, S., \& Yilmaz, E. (2009). Document selection methodologies for efficient and effective learning-to-rank. In Proceedings of the 32nd annual international ACM SIGIR conference on research and development in information retrieval (pp. 468-475). New York, NY: ACM.

Banerjee, S., \& Pedersen, T. (2002). An adapted Lesk algorithm for word sense disambiguation using WordNet. In Lecture notes in computer science: Computational linguistics and intelligent text processing (pp. 136-145). New York: Springer.

Burges, C., Shaked, T., Renshaw, E., Lazier, A., Deeds, M., Hamilton, N., et al. (2005). Learning to rank using gradient descent. In Proceedings of the 22nd international conference on machine learning (pp. 89-96)

Cao, Z., Qin, T., Liu, T., Tsai, M., \& Li, H. (2007). Learning to rank: from pairwise approach to listwise approach. In Proceedings of the 24th international conference on machine learning (pp. 129-136). New York, NY: ACM.

Carterette, B., \& Petkova, D. (2006). Learning a ranking from pairwise preferences. In Proceedings of the 29th annual international ACM SIGIR conference on research and development in information retrieval (pp. 629-630). New York, NY: ACM.

Chakrabarti, S., Khanna, R., Sawant, U., \& Bhattacharyya, C. (2008). Structured learning for non-smooth ranking losses. In Proceedings of the 14th ACM SIGKDD international conference on knowledge discovery and data mining (pp. 88-96). New York, NY: ACM.

Charniak, E. (2000). A maximum-entropy-inspired parser. ACM International Conference Proceedings Series, 4, 132-139.

Cossock, D., \& Zhang, T. (2006) Subset ranking using regression. In Lecture notes in computer science: Learning theory (Vol. 4005, p. 605). New York: Springer.

Denoyer, L., \& Gallinari, P. (2006). The Wikipedia XML corpus. ACM SIGIR Forum, 40(1), 64-69.

Fan, W., Fox, E., Pathak, P., \& Wu, H. (2004). The effects of fitness functions on genetic programmingbased ranking discovery for web search. Journal of the American Society for Information Science and Technology, 55(7), 628-636.

Fellbaum, C. (eds) (1998) WordNet: An electronic lexical database. Cambridge, MA: MIT Press.

Freund, Y., Iyer, R., Schapire, R., \& Singer, Y. (2003). An efficient boosting algorithm for combining preferences. The Journal of Machine Learning Research, 4, 933-969.

Furnkranz, J., \& Hullermeier, E. (2003). Pairwise preference learning and ranking. In Lecture notes in computer science: Machine learning: ECML 2003 (pp. 145-156). New York: Springer.

Goldberg, D., \& Holland, J. (1988). Genetic algorithms and machine learning. Machine Learning, 3(2), 95-99

Herbrich, R., Graepel, T., \& Obermayer, K. (2002). Large margin rank boundaries for ordinal regression. In Proceedings of the 8th ACM SIGKDD international conference on knowledge discovery and data mining (pp. 133-142). New York, NY: ACM.

Higashinaka, R., \& Isozaki, H. (2008). Corpus-based question answering for why-questions. In Proceedings of the international joint conference on natural language processing (IJCNLP) (pp. 418-425).

Hovy, E., Hermjakob, U., \& Ravichandran, D. (2002). A question/answer typology with surface text patterns. In Proceedings of the human language technology conference (HLT), San Diego, CA, USA, pp. 247-251.

Hsu, C., Chang, C., \& Lin, C., et al. (2003). A practical guide to support vector classification. Technical report, Department of Computer Science, National Taiwan University, Taipei 106, Taiwan. 
Japkowicz, N., \& Stephen, S. (2002). The class imbalance problem: A systematic study. Intelligent Data Analysis, 6(5), 429-449.

Joachims, T. (2002) Optimizing search engines using clickthrough data. In Proceedings of the 8th ACM SIGKDD international conference on knowledge discovery and data mining (pp. 133-142). New York, NY: ACM.

Liu, T. (2009). Learning to rank for information retrieval. Foundations and Trends in Information Retrieval, 3(3), 225-331.

Liu, T., Xu, J., Qin, T., Xiong, W., \& Li, H. (2007). Letor: Benchmark dataset for research on learning to rank for information retrieval. In Proceedings of the workshop on learning to rank for information retrieval (LR4IR) at SIGIR 2007 (pp. 3-10).

Maybury, M. (2002). Toward a question answering roadmap. Technical report, Mitre Corporation, Bedford, MA, USA.

Owen, A. (2007). Infinitely imbalanced logistic regression. The Journal of Machine Learning Research, 8, 761-77.

Pedersen, T., Patwardhan, S., \& Michelizzi, J. (2004). WordNet::Similarity-Measuring the relatedness of concepts. In Proceedings of the national conference on artificial intelligence (pp. 1024-1025). Menlo Park: AAAI Press.

Qin, T., Liu, T., Xu, J., \& Li, H. (2008) LETOR: A benchmark collection for learning to rank for information retrieval. Technical report, Microsoft Research Asia

Shen, L., \& Joshi, A. (2005). Ranking and reranking with perceptron. Machine Learning, 60(1), 73-96.

Surdeanu, M., Ciaramita, M., \& Zaragoza, H. (2008). Learning to rank answers on large online QA collections. In Proceedings of the 46th annual meeting on association for computational linguistics (pp. 719-727).

Tang, Y., Zhang, Y., Chawla, N., \& Krasser, S. (2009). SVMs modeling for highly imbalanced classification. IEEE Transactions on Systems, Man, and Cybernetics, Part B, 39(1), 281-288.

Tiedemann, J. (2007). A comparison of genetic algorithms for optimizing linguistically informed IR in question answering. In Lecture notes in computer science: AI*IA 2007: Artificial intelligence and human-oriented computing (Vol. 4733, pp. 398). New York: Springer.

Trotman, A. (2004). An artificial intelligence approach to information retrieval. In Proceedings of the 27th annual international ACM SIGIR conference on research and development in information retrieval (p. 603). New York, NY: ACM.

Usunier, N., Amini, M., \& Gallinari, P. (2004). Boosting weak ranking functions to enhance passage retrieval for question answering. In Proceedings of the workshop on information retrieval for question answering (IR4QA) at SIGIR 2004 (pp. 1-6).

Verberne, S., Boves, L., Oostdijk N., \& Coppen, P. (2007). Evaluating discourse-based answer extraction for why-question answering. In Proceedings of the 30th annual international ACM SIGIR conference on research and development in information retrieval (pp. 735-736). New York, NY: ACM.

Verberne, S., Boves, L., Oostdijk, N., \& Coppen, P. (2008). Using syntactic information for improving whyquestion answering. In Proceedings of the 22nd international conference on computational linguistics (COLING) (pp. 953-960).

Verberne, S., Boves, L., Oostdijk, N., \& Coppen, P. (2010). What is not in the bag of words for why-QA? Computational Linguistics, 32(2), 229-245.

Voorhees, E. (2000). Overview of the TREC-9 question answering track. In Proceedings of TREC-9 (Vol. 7, pp. 1-80).

Voorhees, E. (2003). Overview of the TREC 2003 question answering track. In Proceedings of the twelfth text retrieval conference (TREC 2003) (Vol. 142, pp. 54-68).

Voorhees, E., \& Tice, D. (2000). Building a question answering test collection. In Proceedings of the 23rd annual international ACM SIGIR conference on research and development in information retrieval (pp. 200-207). New York, NY: ACM.

Xia, F., Liu, T., Wang, J., Zhang, W., \& Li, H. (2008). Listwise approach to learning to rank: Theory and algorithm. In Proceedings of the 25th international conference on machine learning (pp. 1192-1199). New York, NY: ACM

Xu, J., \& Li, H. (2007) Adarank: A boosting algorithm for information retrieval. In Proceedings of the 30th annual international ACM SIGIR conference on research and development in information retrieval (pp. 391-398). New York, NY: ACM.

Yeh, J., Lin, J., Ke, H., Yang, W. (2007). Learning to rank for information retrieval using genetic programming. In Proceedings of the workshop on learning to rank for information retrieval (LR4IR) at SIGIR 2007. 
Yue, Y., Finley, T., Radlinski, F., \& Joachims, T. (2007). A support vector method for optimizing average precision. In Proceedings of the 30th annual international ACM SIGIR conference on research and development in information retrieval (pp. 271-278). New York, NY: ACM.

Zhai, C. (2001). Notes on the Lemur TFIDF model. Technical report, School of Computer Science, Carnegie Mellon University. 